\title{
Does phone messaging improves tuberculosis treatment success? A systematic review and meta-analysis
}

Kassahun Dessie Gashu 1* (D, Kassahun Alemu Gelaye², Zeleke Abebaw Mekonnen', Richard Lester ${ }^{3}$ and Binyam Tilahun ${ }^{1,3}$

\begin{abstract}
Background: Compliance to anti-TB treatment is crucial in achieving cure and avoiding the emergence of drug resistance. Electronic health (eHealth) interventions are included in the strategy to end the global Tuberculosis (TB) epidemic by 2035. Evidences showed that mobile messaging systems could improve patient adherence to clinic appointment for diagnosis and treatment. This review aimed to assess the effect of mobile-phone messaging on anti-TB treatment success.

Methods: All randomized controlled trial (RCT) and quasi-experimental studies done prior to August 26, 2019 were included in the review. Studies were retrieved from PubMed, EMBASE, Cochrane and ScienceDirect databases including, grey and non-indexed literatures from Google and Google scholar. Quality of studies were independently assessed using Cochrane Risk of Bias Assessment Tool. A qualitative synthesis and quantitative pooled estimation were used to measure the effect of phone messaging on TB treatment success rate. PRISMA flow diagrams were used to summarize article selection process.

Results: A total of 1237 articles were identified, with 14 meeting the eligibility criteria for qualitative synthesis. Eight studies with a total of 5680 TB patients (2733 in intervention and 2947 in control groups) were included in metaanalysis. The pooled effect of mobile-phone messaging revealed a small increase in treatment success compared to standard of care (RR 1.04, 95\% Cl 1.02 to 1.06), with low heterogeneity $\left(I^{2}=7 \%, p<0.0002\right)$. In the review, performance, detection and attrition biases were reported as major risk of biases.

Conclusions: Mobile-phone messaging showed a modest effect in improving anti-TB treatment success; however, the quality of evidence was low. Further controlled studies are needed to increase the evidence-base on the role of mHealth interventions to improve TB care.
\end{abstract}

Protocol registration number: CRD420170744339. http://www.crd.york.ac.uk/PROSPERO/display_record.php?ID=CRD42 017074439

Keywords: Mobile phone, Treatment success, Text messaging, Tuberculosis

\footnotetext{
* Correspondence: kassahundessie@gmail.com

${ }^{1}$ Department of Health Informatics, Institute of Public Health, College of

Medicine and Health Sciences, University of Gondar, Gondar, Ethiopia

Full list of author information is available at the end of the article
}

C The Author(s). 2020 Open Access This article is distributed under the terms of the Creative Commons Attribution 4.0 International License (http://creativecommons.org/licenses/by/4.0/), which permits unrestricted use, distribution, and reproduction in any medium, provided you give appropriate credit to the original author(s) and the source, provide a link to the Creative Commons license, and indicate if changes were made. The Creative Commons Public Domain Dedication waiver (http://creativecommons.org/publicdomain/zero/1.0/) applies to the data made available in this article, unless otherwise stated. 


\section{Background}

Globally, mobile cellular subscription coverage is growing exponentially [1]. The unprecedented spread and advancements in innovative application of mobile technologies is an opportunity to address health priorities [2]. Some mobile health applications have shown improvement in patients' adherence to medications [3]. Evidences demonstrated that mobile-phone text messaging were effective in ART programs [4-7]. Electronic Health interventions has now brought attention in the strategy to end the global TB epidemic by 2035 [8]. In 2015, World Health Organization (WHO) established its Global Task Force on Digital Health for TB to advocate and support the development of digital health innovations in global efforts to improve TB care and prevention [9]. Electronic based patient education was components of a WHO conceptual framework for digital health in the TB response. This was intended to be accomplished using video (virtually) observed therapy [10], Short Message Service (SMS) and eLearning [11].

Tuberculosis is one of the critical public health threat which is the leading cause of mortality worldwide [12]. WHO estimated that globally one-third of the world population harbors latent TB infections [13]. An estimated 10 million TB cases in 2017 and the 30 High Burden Countries (HBCs) accounted about $90 \%$ of the global TB burden since 2015 [14].

Compliance to anti-TB treatment is crucial in achieving cure and avoiding the emergence of drug resistance (MDR- and XDR-TB).

Regular and complete medication intake gives individual TB patients the best chance of cure and also protects the community from the spread of TB [15]. One untreated infectious tuberculosis patient is likely to infect 10 to 15 persons annually [16]. The consequences of poor adherence to long-term therapies are poor health outcomes and increased health care costs interventions aimed at improving adherence would provide a significant positive return on investment through primary prevention and secondary prevention of adverse health outcomes [17-22].

Evidences showed that mobile messaging systems could improve patient adherence to clinic appointment for diagnosis and treatment [23]; $\mathrm{TB}$ prevention and promotion of Anti-TB treatment adherence [24, 25]. But evidences were limited on effect of phone messaging intervention specifically on Anti-TB treatment success. According to WHO, Anti-TB treatment success is defined as the sum of cured and treatment completed. The cure rate is defined as the percentage of patients who completed treatment and were culture negative during the last month of treatment and on at least one other occasion for non-MDR-TB and who had at least 5 consecutive results in the previous $12-15$ months for MDRTB. Whereas, completion rate is the percentage of
MDR-TB and non-MDR-TB patients who completed treatment according to guidelines but did not meet the definition for cure or treatment failure due to a lack of bacteriological results [26]. According to the latest treatment outcome, the global treatment success rate was $82 \%$ in 2016 that showed a reduction from $86 \%$ in 2013 and $83 \%$ in 2015 [27].

The output of this review could be useful evidence for policy makers, researchers, care providers, community members and patients to consider in due plan and implementations. This systematic review was aimed:

* To appraise existing evidences of SMS to improve Anti-TB Treatment Success Rate (TSR)

* To assess pooled effect of SMS to improve Anti-TB TSR

* To assess the effect of frequency of messaging to improve TSR

* To identify type of SMS (reminder/educational messaging) more important for TSR.

\section{Methods}

The protocol has been registered at PROSPERO with ID: CRD420170744339; http://www.crd.york.ac.uk/ PROSPERO/display_record.php? ID = CRD42017074439.

\section{Criteria for considering studies for this review Types of study}

This study included RCT and Quasi-experimental studies comparing DOTS with and without mobile-phone reminder for patients on Anti-TB treatment. Studies written only in English were include in the review. Studies that measured treatment success including treatment completeness and cure were included in the in the qualitative synthesis. For meta-analysis, we included studies reported treatment success rate and studies reported data elements that enabled us to calculate treatment success rate.

\section{Types of participants}

TB patients aged $\geq 15$ years were included in the review.

\section{Types of interventions}

Studies that used mobile-phone based messaging service for patients on DOTS as interventions group and those patients on DOTS without messaging was control groups.

\section{Information source and search strategies}

In this systematic review, databases including PubMed, EMBASE, Cochrane, and ScienceDirect were searched. In addition, grey literature and non-indexed articles were searched on Google and Google scholar. Searching strategies were based on Population, Intervention, Comparison, and 
Outcome (PICO) criteria and using Medical Subject Headings $(\mathrm{MeSH})$, Boolean search operators, truncation symbols to robust the searching scheme. Searching was held for articles disseminated up to August 26, 2019. Please see the $\mathrm{MeSH}$ searching terms (Additional file 1).

\section{Study selection}

Searched results were exported to Endnote X7 citation manager, duplicates were removed and studies were examined based on selection criteria. Abstracts of studies were examined for eligibility for meta-analysis and the full text of articles was searched when abstracts did not provide sufficient information to make a decision. Searching was independently carried out by two reviewers (KDG and ZAM) to reduce selection bias.

\section{Data collection process}

In this review, data collection and analysis were based on the Cochrane Handbook of Systematic Reviews for Interventions [28].

\section{Risk of bias assessment}

Quality of studies were independently assessed by two authors (KDG and ZAM) using Cochrane Risk of Bias Assessment Tool including: use of random sequence generation; concealment of allocation to conditions; blinding of participant and personnel; blinding of outcome assessors; completeness of outcome data and other; selective reporting and other biases. Each study was rated as low risk of bias when there was no concern regarding bias; as high risk of bias when there was concern regarding bias; or unclear risk of bias if the

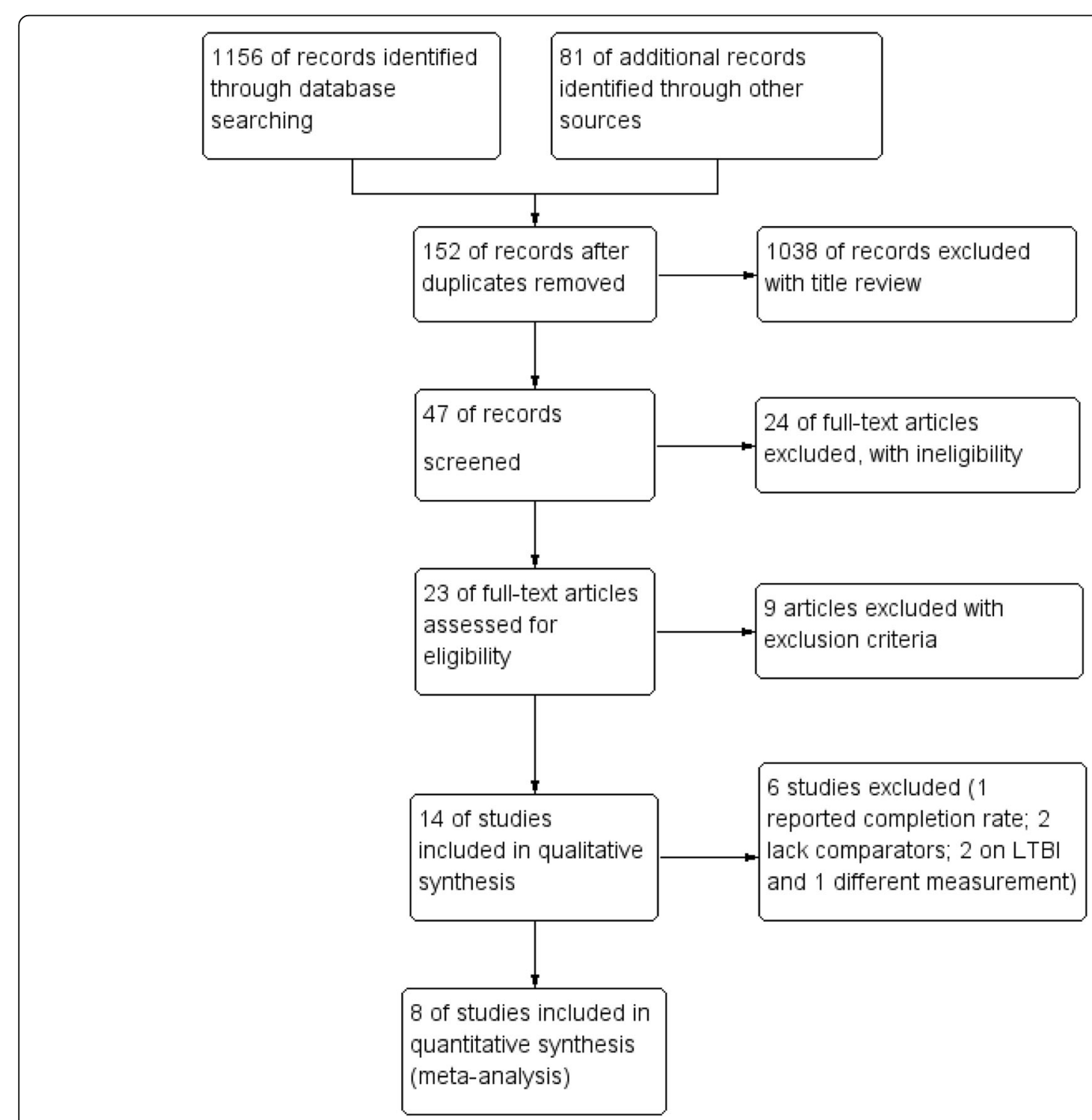

Fig. 1 PRISMA flow diagram for inclusion of articles 
information was absent [28]. The level of agreement between the two reviewers were measured using Cohen's Kappa level of agreement [29]. Third body was planned to decide if the two reviews couldn't able to agree on a certain point of bias assessment.

\section{Data extraction and analysis}

All shortlisted articles were independently reviewed by two reviewers and decided to include in the systematic review and then to the meta-analysis. Both authors (KDG and ZAM) independently extracted the selected measures of Anti-TB treatment success. Cure and treatment completeness were directly used for measuring $\mathrm{TB}$ treatment success. Treatment success was also indirectly estimated from unsuccessful treatment outcomes like, death, loss to follow-up, treatment failure and transferout. Cochrane Collaboration Review Manager Version 5.3 software was used for data extraction and analysis.

\section{Effect size determination}

Anti-TB treatment success rate is measured by adding patients cured and those completed their treatment [26]. The pooled effects of intervention (mobile-phone messaging) was measured by using risk ratios (RR) and with 95\% confidence intervals. The statistical heterogeneity between studies and its impact on meta-analysis was examined using the $\mathrm{I}^{2}$ statistic. Where, $\mathrm{I}^{2}(0 \%=$ no, $25 \%=$ low, $50 \%=$ moderate, $75 \%=$ high heterogeneity of effect sizes) [28]. A fixed and random effect models were used to estimate the RR $(95 \% \mathrm{CI})$ based on the level of heterogeneity $\left(\mathrm{I}^{2}\right)$ of the included studies [30,31]. Sensitivity and subgroup analyses were used to identify the potential effect of heterogeneity of studies was evaluated using.

\section{Sensitivity analysis}

Effect size analysis was run with and without the outlier to assess its effect on the overall findings. To assess whether individual studies had impact on summary effect, summary analysis was undertaken after removing each studies effect [28].

\section{Subgroup analysis}

Four subgroups were pre-determined based on a priori chosen sub-variables aimed to understand their independent contributions in enhancing mobile-phone messaging to improve Anti-TB treatment success. These sub-variables were: purpose of messaging which classified as medication reminder and educational messaging; disease burden which classified as High Burden Countries (HBCs) and Non-HBCs; type of mobile-phone messaging used (Text, call, graphic reminder, etc.) and frequency of messaging (Daily, Weekly \& Both Daily \& Weekly).

\section{Assessment of publication bias}

Publication bias was assessed using a funnel plot. When asymmetry was indicated by the funnel plot of the effect sizes by their standard error, the impact on the summary effect size was assessed. Egger's test was used to see statistical test of publication bias. When an outlier was detected, the relevant study was re-examined.

\section{Rating quality of evidence}

The Grading of Recommendations Assessment, Development, and Evaluation (GRADE) system was used to rate the quality of evidence of this review. Five factors, namely; limitations, inconsistency, indirectness, imprecision, and publication bias were used to rate the quality of evidence [32].

\section{Results}

Selection of studies

Articles were retrieved from databases including PubMed (552), EMBASE (344), ScienceDirect (168), Cochrane (92) and grey literature from Google Scholar (78) and reference lists (3), as shown in the flow diagram for selection processes (Fig. 1).

From the total of 1237 articles retrieved, 152 articles excluded due to duplication; 1039 excluded with title review and 24 articles excluded due to ineligibility. Finally, 23 articles were fully read and 9 studies were excluded from the review with different reasons, as shown in Table 1. Some articles were excluded because of using video-based directly observed therapy [33-37] the intervention was different from phone text, audio, graphic and video messages. SMS intervention has an unrelated

Table 1 Characteristics of excluded studies

\begin{tabular}{ll}
\hline Study & Reasons for exclusion \\
\hline $\begin{array}{l}\text { Garfein, et'al, } \\
2015 \text { [33] }\end{array}$ & $\begin{array}{l}\text { Different intervention (Video Based Directly } \\
\text { Observed Therapy) }\end{array}$ \\
$\begin{array}{l}\text { Holzschuh, et'al, } \\
2017 \text { [34] }\end{array}$ & Video Based Directly Observed Therapy \\
Buchman, et'al, & Different intervention (Skype Observed Therapy) \\
2017 [35] & Remote (video) mobile Direct Observation of \\
Hoffman, et'al, & Treatment \\
2010 [36] & Focus on videophone technology \\
$\begin{array}{l}\text { DeMaio, et'al, } \\
2001 \text { [37] }\end{array}$ & SMS was used for issuing of test results not for \\
Lorent, et'al, & treatment reminder \\
2014 [38] & Study subjects were HIV/TB co-infected patients \\
Bassett, et'al, & \\
2016 [39] & Intervention for Health care workers \\
Chaiyachati, et'al, & \\
2013 [5] & Participants were HIV/TB co-infected \\
Howard, et'al, & \\
2016 [40] & \\
\hline
\end{tabular}




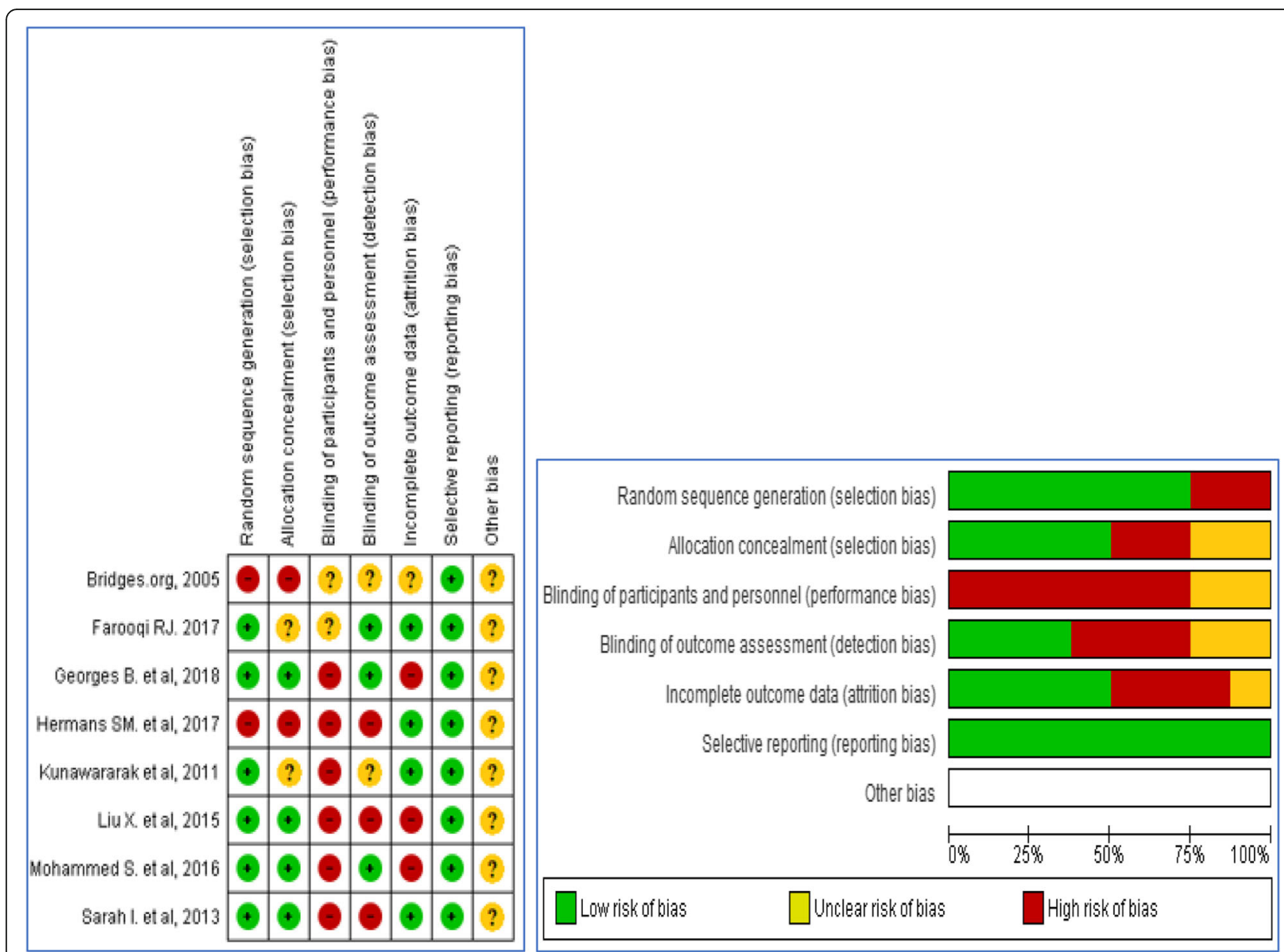

Fig. 2 Risk of bias summary and graph: authors' judgments for each included study

\begin{tabular}{|c|c|c|c|c|c|c|c|c|c|c|}
\hline Study or Subgroup & $\begin{array}{c}\text { SMS } \\
\text { Events }\end{array}$ & Total & $\begin{array}{l}\text { Non-S } \\
\text { Events }\end{array}$ & $\begin{array}{l}\text { MS } \\
\text { Total }\end{array}$ & Weight & $\begin{array}{c}\text { Risk Ratio } \\
\text { M-H, Fixed, } 95 \% \mathrm{Cl}\end{array}$ & & $\begin{array}{r}\text { Risk I } \\
\text { M-H, Fixe }\end{array}$ & $\begin{array}{l}\text { Ratio } \\
\text { d, 95\% Cl }\end{array}$ & \\
\hline Bridges org, 2005 & 161 & 221 & 153 & 221 & $6.4 \%$ & $1.05[0.93,1.19]$ & & & & \\
\hline Faroogi RJ. 2017 & 70 & 74 & 69 & 74 & $29 \%$ & $1.01[0.93,1.10]$ & & & & \\
\hline Georges B. et al, 2018 & 111 & 137 & 106 & 142 & $4.4 \%$ & $1.09[0.96,1.23]$ & & & & \\
\hline Hermans SM et al, 2017 & 171 & 183 & 268 & 302 & $8.5 \%$ & $1.05[1.00,1.11]$ & & & & \\
\hline Kunawararak et al, 2011 & 30 & 30 & 29 & 30 & $1.2 \%$ & $1.03[0.94,1.13]$ & & . & & \\
\hline LiuX et al, 2015 & 913 & 966 & 945 & 1066 & $37.7 \%$ & $1.07[1.04,1.09]$ & & & + & \\
\hline Mohammed 8 e et al, 2016 & 917 & 1104 & 903 & 1093 & $38.1 \%$ & $1.01[0.97,1.04]$ & & & & \\
\hline Sarah l. et al, 2013 & 17 & 18 & 17 & 19 & $0.7 \%$ & $1.06[0.87,1.28]$ & & & & \\
\hline Total (95\% Cl) & & 2733 & & 2947 & $100.0 \%$ & $1.04[1.02,1.06]$ & & & $\boldsymbol{\varphi}$ & \\
\hline Total events & 2390 & & 2490 & & & & & & & \\
\hline \multicolumn{7}{|c|}{$\begin{array}{l}\text { Heterogeneity: } C h i^{2}=7.54, \mathrm{~d}=7(\mathrm{P}=0.37) ; 1^{2}=7 \% \\
\text { Test for owerall effect: } \mathrm{Z}=3.71(\mathrm{P}=0.0002)\end{array}$} & 0.7 & $\begin{array}{l}0.85 \\
\text { None SMS }\end{array}$ & SMS groups & 1.5 \\
\hline
\end{tabular}


purpose [38] and study participants were not matching with this review $[5,39,40]$.

\section{Characteristics of selected studies}

After exclusion of ineligible studies, fourteen studies were selected and reviewed for the qualitative synthesis of evidence. Among included studies, four in Africa, seven in Asia, one in South in one in North America, and one study enrolled patients from North America, Europe, Asia, and Africa. See details in Table 3 in the Appendix.

All studies implemented mobile phone messaging interventions on top of the standard TB treatment and care. The various types of phone messaging interventions were applied. Nine of fourteen studies used textbased phone messaging, two studies used only phone call and two studies applied a text and/or voice messages. One study implemented text and graphic messages for $\mathrm{TB}$ patients to adhere to their treatment.
Among all studies, the messaging intervention was interactive (two-way) in seven studies, one-way in five and two studies didn't report on the messaging model. The ultimate purpose of mobile messaging in 11 studies were to remind patients towards their anti-TB medication. In 3 studies the messaging intervention aimed to identify patients' concerns, educate and motivate patients to engage on their own medication.

A study in Thailand [41] reported a significant effect of SMS messaging on TB treatment success rate. Whereas, other randomized control study in Argentina [42], in Pakistan [43, 44], in China [45] found no significant difference between the SMS or control groups for treatment success.

Two studies didn't calculate significance test on the effect of SMS messaging on TSR of TB due to inappropriate or lack of comparator group [46, 47]. One study reported only about the level of patient adherence on TB treatment

\begin{tabular}{|c|c|c|c|c|c|c|c|c|c|c|}
\hline Study or Subgroup & $\begin{array}{r}\text { SMS } \\
\text { Events }\end{array}$ & S Total & \multicolumn{2}{|c|}{ Non-SMS } & Weight & $\begin{array}{c}\text { Risk Ratio } \\
\text { M-H, Fixed, } 95 \% \mathrm{Cl}\end{array}$ & \multicolumn{4}{|c|}{$\begin{array}{c}\text { Risk Ratio } \\
\text { M-H, Fixed, } 95 \% \mathrm{Cl}\end{array}$} \\
\hline Bridges.org, 2005 & 161 & 221 & 153 & 221 & $10.4 \%$ & $1.05[0.93,1.19]$ & & & 1 & \\
\hline Faroogi R.J. 2017 & 70 & 74 & 69 & 74 & $4.7 \%$ & $1.01[0.93,1.10]$ & & & & \\
\hline Georges B. et al, 2018 & 111 & 137 & 106 & 142 & $7.1 \%$ & $1.09[0.96,1.23]$ & & & & \\
\hline Hermans SM. et al, 2017 & 171 & 183 & 268 & 302 & $13.7 \%$ & $1.05[1.00,1.11]$ & & & $\rightarrow$ & \\
\hline Kunawararak et al, 2011 & 30 & 30 & 29 & 30 & $2.0 \%$ & $1.03[0.94,1.13]$ & & & & \\
\hline LiuX. et al, 2015 & 913 & 966 & 945 & 1066 & $61.0 \%$ & $1.07[1.04,1.09]$ & & & 7 & \\
\hline Wohammed S. et al, 2016 & 917 & 1104 & 903 & 1093 & $0.0 \%$ & $1.01[0.97,1.04]$ & & & & \\
\hline Sarah I. et al, 2013 & 17 & 18 & 17 & 19 & $1.1 \%$ & $1.06[0.87,1.28]$ & & & & \\
\hline Total $(95 \% \mathrm{Cl})$ & & 1629 & & 1854 & $4100.0 \%$ & $1.06[1.04,1.09]$ & & & 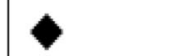 & \\
\hline Total events & 1473 & & 1587 & & & & & & & \\
\hline $\begin{array}{l}\text { Heterogeneity: } \mathrm{Ch}^{2}=1.81 \text {, } \\
\text { Test for overall effect: } Z=4\end{array}$ & $\begin{array}{l}d f=6(P= \\
86(P<0.1\end{array}$ & $\begin{array}{l}=0.94) ; \\
00001)\end{array}$ & $1^{2}=0 \%$ & & & & 0.7 & $\begin{array}{l}0.85 \\
\text { None SMS }\end{array}$ & $\begin{array}{rr}1.2 \\
\text { SMS groups }\end{array}$ & 1.5 \\
\hline Study or Subgroup & $\begin{array}{l}\text { SMS } \\
\text { Events }\end{array}$ & Total & $\begin{array}{l}\text { Non-SM } \\
\text { Events }\end{array}$ & $\begin{array}{l}\text { MS } \\
\text { Total }\end{array}$ & Weight & $\begin{array}{c}\text { Risk Ratio } \\
\text { M-H, Random, } 95 \% \mathrm{Cl}\end{array}$ & & $\begin{array}{r}\text { Risk } \\
\text { M-H, Rand }\end{array}$ & $\begin{array}{l}\text { Ratio } \\
\text { dom, } 95 \% \mathrm{Cl}\end{array}$ & \\
\hline Bridges org, 2005 & 161 & 221 & 153 & 221 & $4.9 \%$ & $1.05[0.93,1.19]$ & & 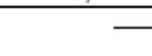 & & \\
\hline Faroogi RJ. 2017 & 70 & 74 & 69 & 74 & $10.3 \%$ & $1.01[0.93,1.10]$ & & & & \\
\hline Georges B. et al, 2018 & 111 & 137 & 106 & 142 & $4.4 \%$ & $1.09[0.96,1.23]$ & & & & \\
\hline Hermans SM. et al, 2017 & 171 & 183 & 268 & 302 & $22.4 \%$ & $1.05[1.00,1.11]$ & & & $\rightarrow-$ & \\
\hline Kunawararak et al, 2011 & 30 & 30 & 29 & 30 & $8.3 \%$ & $1.03[0.94,1.13]$ & & & & \\
\hline LiuX. et al, 2015 & 913 & 966 & 945 & 1066 & $0.0 \%$ & $1.07[1.04,1.09]$ & & & & \\
\hline Wohammed S. et al, 2016 & 917 & 1104 & 903 & 1093 & $47.8 \%$ & $1.01[0.97,1.04]$ & & & - & \\
\hline Sarah l. et al, 2013 & 17 & 18 & 17 & 19 & $1.9 \%$ & $1.06[0.87,1.28]$ & & & & \\
\hline Total (95\% Cl) & & 1767 & & 1881 & $100.0 \%$ & $1.03[1.00,1.05]$ & & & $>$ & \\
\hline Total events & 1477 & & 1545 & & & & & & & \\
\hline $\begin{array}{l}\text { Heterogeneity: Tau }{ }^{2}=0.00 \text {; } \\
\text { Test for overall effect } Z=1\end{array}$ & $\begin{array}{l}\mathrm{Chi}^{2}=3.08 \\
80(\mathrm{P}=0.0\end{array}$ & $\begin{array}{l}8, d f=6 \\
\text { (6) }\end{array}$ & $6(P=0.80$ & $(0) ; 1^{2}=$ & & & 0.7 & $\begin{array}{l}.85 \\
\text { None SMS }\end{array}$ & $1 \frac{1.2}{\text { SMS groups }}$ & 1.5 \\
\hline
\end{tabular}

Fig. 4 Sensitivity analysis excluding Mohammed et al. and Liu X. et al. studies to see its influence on effect size estimation 
but not on treatment outcome [48]. The characteristics of each selected studies have explained in Table 3. Two randomized controlled studies in USA, Spain, Hong Kong and South Africa [49], and in Canada [52], reported that phone messaging did not significantly improve LTBI completion rates compared to standard care. Successful treatment of Latent Tuberculosis Infection (LTBI) also plays key role in eliminating TB [55]; however, difficult to ascertain treatment success in LTBI cases.

\section{Quantitative synthesis of evidence}

Only eight studies $[41-45,50,53,54]$ were found to be fitted for the pooled estimation of the effect of mobile phone messaging on successful TB treatment outcome. Six studies were excluded with the following reasons. One study reported treatment completion and unable to calculate the treatment success rate from the reported data [51]. Two studies focused on LTBI treatment completion $[49,52]$ couldn't be pooled to determining treatment success. One study used a different tool to measure the outcome variable [48] and studies excluded due to lack of comparison groups $[46,47]$.
Risk of bias assessment for the included studies

Among the selected studies for meta-analysis, performance bias was the major challenge in the studies, because of the nature of the intervention, participants could not be blinded. Blinding of outcome assessors was also major gap that could lead to detection bias. All studies were jugged free of selection and reporting bias. Two studies have attrition bias (Fig. 2). Based on Cohen's Kappa level of agreement, the two reviewers (KDG and ZAM) have shown $83.3 \%$ agreement with $\mathrm{k}=0.686, p<0.0001$ for the included papers.

\section{Pooled estimation on the effect of phone messaging on TB treatment success}

Overall, 5680 TB patients, 2733 in intervention and 2947 in control groups were involved in the pooled analysis. The overall Anti-TB treatment success was 2390/2733 (87.4\%) in intervention and 2490/2947 (84.5\%) in control groups with heterogeneity level $\left(\mathrm{I}^{2}=7 \%, p<0.0002\right)$. Fixed-effect model has shown that phone messaging group had higher treatment success rate compared to

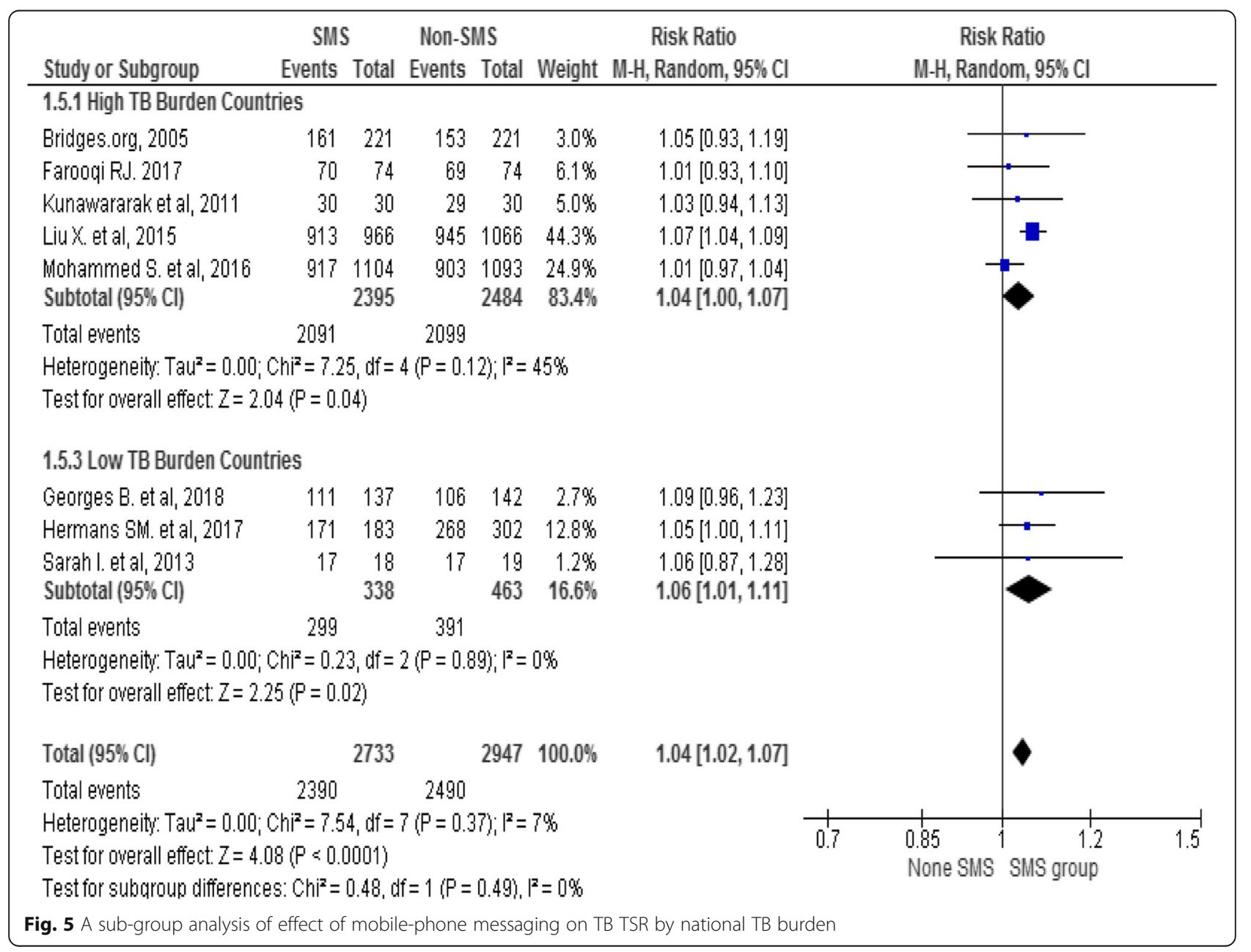


standard care (RR 1.04, 95\% CI 1.02 to 1.06), see the Forest plot in Fig. 3.

Sensitivity analysis was carried out to see the effect size of each model by excluding every one of the studies. All studies were taken out of the analysis one by one and the output indicated that no single study separately influenced the pooled effect of phone messaging on TB treatment success. The Forest plot for sensitivity analysis for the two big weighted studies (Mohammed et al. and Liu et al) presented on Fig. 4.

\section{Sub-group analysis}

Sub-group analysis was carried out to identify the effect of interventions by the level of national TB burden (high Vs low) and model of phone messaging (one-way Vs two-way) applied by individual studies. The finding indicated that phone messaging has a modest effect on TSR in high TB burden ( $\mathrm{RR}=1.04 ; 95 \% \mathrm{CI} 1.00-1.07)$ and in low TB burden countries ( $R R=1.06$; 95\% CI 1.01-1.11) with moderate $\left(\mathrm{I}^{2}=53 \%\right)$ and no $\left(\mathrm{I}^{2}=0 \%\right)$ heterogeneity of studies in both groups respectively see Fig. 5 .

Similarly, a sub-group analysis by model of phone messaging (one-way Vs two-way) shown that interactive phone messaging has modest effect on TSR of TB (Fig. 6). Further sub-group analyses (purpose of the messaging, frequency of messaging, type of messaging and national income) were not conducted due to unable to get sufficient studies in the pre-determined sub-groups.

\section{Publication bias}

A subjective visual inspection of funnel plot of the included studies pointed out symmetrical observation that shows less likely occurrence of publication bias. We used Egger's test to objectively measured publication bias. The finding revealed that there was no evidence of publication bias $(P=0.753)$, (Fig. 7).

\section{Rating quality of evidence}

Based on GRADE quality of evidence assessment approach, the overall quality of evidence of this review was rated low, mainly due to limitations of performance, detection and selection biases for more details see Table 2 .

\section{Discussion}

The substantial growth of mobile phones throughout the world has brought opportunities for integrating mobile

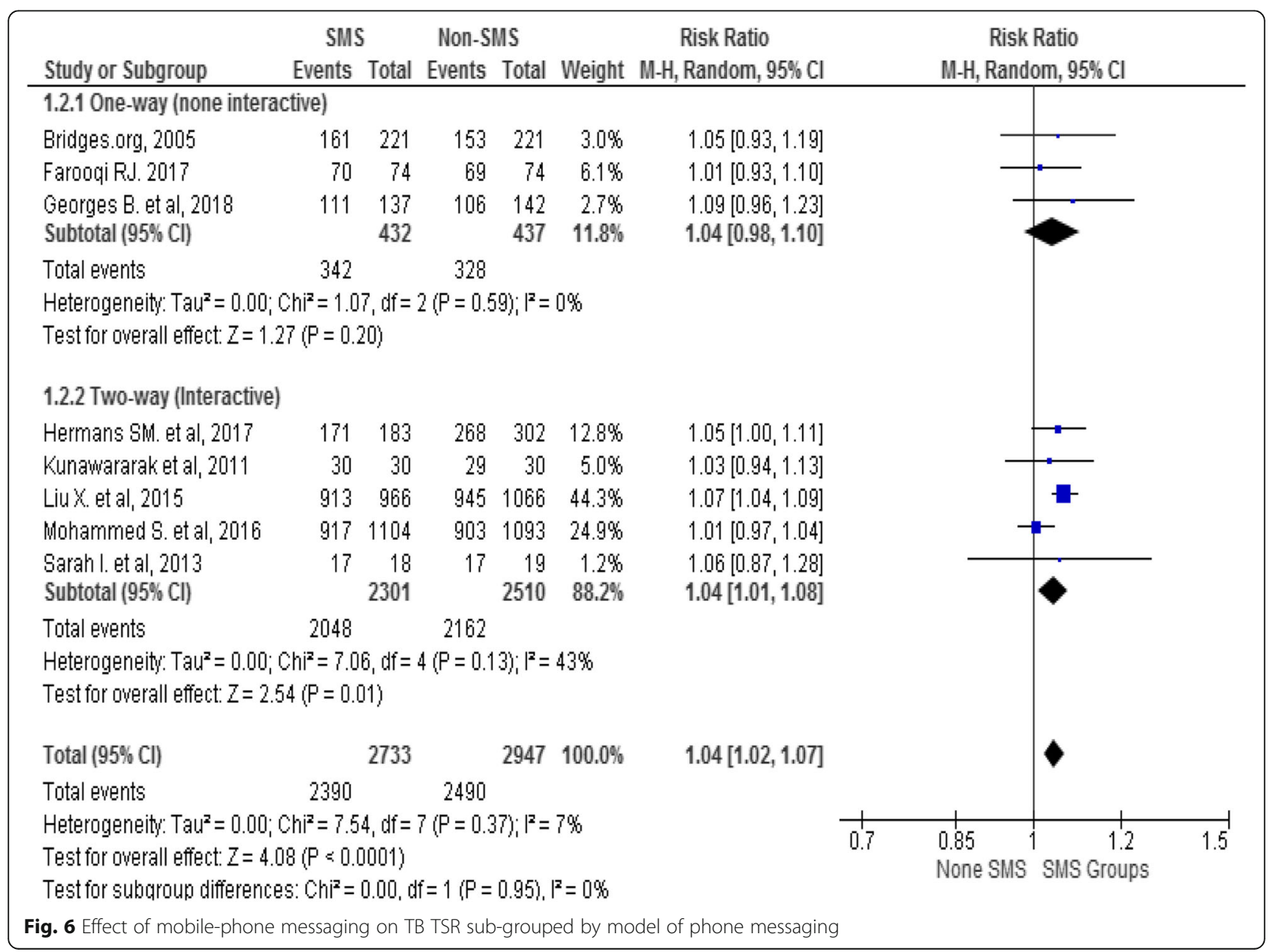




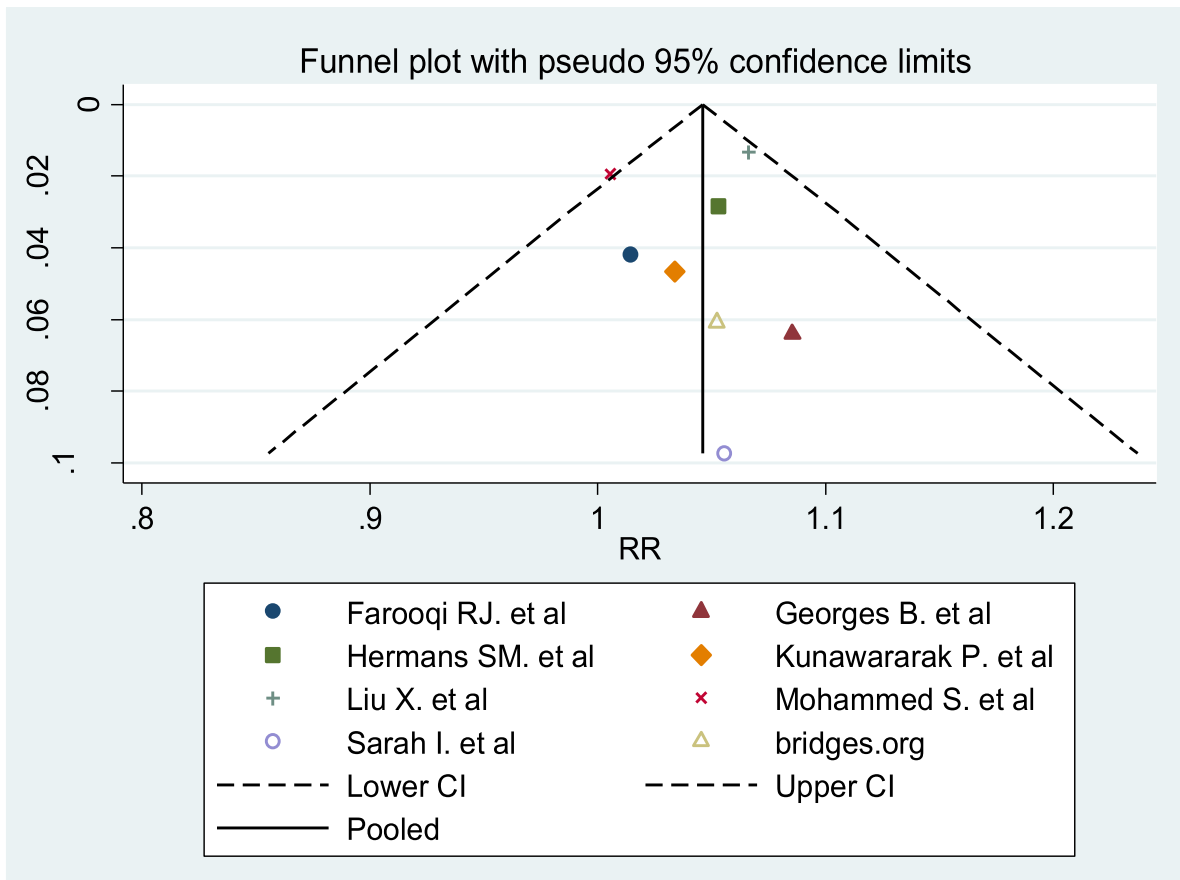

Fig. 7 Funnel Plot of studies comparing phone messaging and standard of care on TSR of TB

phones as a health care intervention tools for TB patients [56]. Mobile Health is now inevitable to play big role to end the global TB epidemic [57]. In this review majority of the studies were obtained from Asia followed by Africa. About 64\% (9/14) the studies implemented text-based phone messaging. Seven (50\%) of the studies used interactive (two-way) approach.

In the current review, the pooled treatment success rate of TB was higher $87.4 \%$ in the intervention group than $84.5 \%$ in the control group. The finding conveyed slight growth from the latest global treatment success rate of $82 \%$ in 2016 [27]. The variation could be due to the recent studies that included the review. The meta-analysis has shown that phone messaging group had a modest increment in treatment success rate compared to standard care (RR 1.04, 95\% CI 1.02 to 1.06) with low heterogeneity ( $\left.\mathrm{I}^{2}=7 \%, p<0.0002\right)$ between individual studies based on the cutoff value [28]. Similar systematic reviews showed that text-messaging interventions were effective for self-management of diabetes, weight loss, smoking cessation, physical activities and medication adherence for antiretroviral therapy $[58,59]$. In the current review, the effect size was low as compared to evidence on ART. The variation could be mHealth has been well studied on ART. Whereas, evidence on the effect of mHealth on TB treatment remains limited [60-62]. The result implies that mobile-phone messaging has a promising effect in improving Anti-TB treatment success; however, further implementation science studies may require to endorse impact, usability and sustainability of mHealth interventions on supporting the global TB epidemic.

In this review, a sub-group analysis indicated that phone messaging has a modest effect on TB treatment success rate in both high and low TB burden countries. The impact could be from the growing penetration of mobile phone technology and mHealth initiatives globally including high disease burden and resource limited countries $[56,57]$. The subgroup

Table 2 GRADE rating of the quality of evidence

\begin{tabular}{|c|c|c|c|c|c|}
\hline Outcomes & $\begin{array}{l}\text { № of participants } \\
\text { (studies) Follow up }\end{array}$ & $\begin{array}{l}\text { Certainty of the } \\
\text { evidence (GRADE) }\end{array}$ & $\begin{array}{l}\text { Relative effect } \\
(95 \% \mathrm{Cl})\end{array}$ & $\begin{array}{l}\text { Anticipated absolut } \\
\text { effects }^{\mathrm{a}}(95 \% \mathrm{Cl})\end{array}$ & \\
\hline \multirow{3}{*}{$\begin{array}{l}\text { TB treatment success } \\
\text { assessed with: } \\
6-9 \text { months }\end{array}$} & \multirow[t]{3}{*}{5680 (8 Studies) } & \multirow[t]{3}{*}{$\oplus \oplus \ominus \ominus \mathrm{LOW}^{\mathrm{b}, c}$} & \multirow[t]{3}{*}{ RR 1.04 (1.02 to 1.06$)$} & Assumed risk & \\
\hline & & & & Usual care group & Risk difference with phone Messaging \\
\hline & & & & 901 per 1000 & 36 more per 1000 ( 18 more to 54 more) \\
\hline
\end{tabular}

${ }^{a}$ The risk in the intervention group (and its $95 \%$ confidence interval) is based on the assumed risk in the comparison group and the relative effect of the intervention (and its $95 \% \mathrm{Cl}$ ). Cl Confidence interval, RR Risk ratio

bHigh performance bias, detection and selection biases

'Some studies used self-assessment tools that subjectively measure treatment completeness 


\section{Appendix}

Table $\mathbf{3}$ Characteristics studies included in the review

\begin{tabular}{|c|c|}
\hline Study ID & Belknap, et al, 2017 [49] \\
\hline Setting & USA, Spain, Hong Kong and South Africa \\
\hline Methods & A randomized clinical trial \\
\hline Participants & Adults (aged $\geq 18$ years) LTBI cases \\
\hline Sample size & Overall, 1002 LTBI case were participated \\
\hline Interventions & A one-way weekly text-based treatment reminder \\
\hline Outcomes & $\begin{array}{l}\text { Treatment completion was } 87 \% \text { in DOTS, } 74 \% \text { in self- } \\
\text { administration and } 76 \text { in self-administration plus SMS re- } \\
\text { minders groups. }\end{array}$ \\
\hline
\end{tabular}

Limitations All participants were not randomly assigned to receive SMS reminders

Study ID Barclay, 2009 [46]

Setting South Africa

Methods A single arm interventional study from July, 2006 to April, 2007

Participants Tuberculosis patients at three clinics in the Cape Town

Sample size 155 patients

Interventions Text messaging using SIMpill for 10 months

Outcomes Drug adherence stabilized between 86 and 92\% with a treatment success rate of $94 \%$ after patients used the SIMpill for 10 months.

Limitations No comparator groups

Study ID ～bridges.org, 2005 [50]

Setting South Africa

Methods A single arm trial with historical control

Participants All adults with pulmonary and extra pulmonary TB were included in the study

Sample size About $221 \mathrm{~TB}$ patients were followed in single arm design

Interventions A one-way text-based daily phone reminder for anti-TB medication for six and eight months

Outcomes Treatment success rate was 73\% in trial and 69\% in the latest statistics available for the City of Cape Town's TB Control Program

Limitations No inferential statistics in treatment success rates, due to limited sample size

Study ID Fang X.H. et al, 2017 [51]

Setting China

Methods A Randomized controlled trial was conducted from December 1, 2014 to 31, 2015

Participants All pulmonary TB patients from six districts

Sample size Overall 350 (160 in intervention and 190 in control groups)

Interventions A one-way text-based daily phone reminder for anti-TB medication for six months

Outcomes The treatment completion rate in SMS group (96.25\%) was significantly higher than that in the control group (86.84\%), p-0.002

Limitations Study included few predictor variables and generalizability restricted to one province
Table 3 Characteristics studies included in the review

(Continued)

Study ID Farooqi et'al, 2015 [44]

Setting Pakistan

Methods Randomized controlled trial was conducted from June 2014 to June 2015

Participants Patients enrolled for anti-TB drugs were distributed in intervention and control groups

Sample size 148 TB patients

Interventions A one-way text and graphic reminders sent daily to intervention group for two months

Outcomes Primary outcome was default, defined as not taking medicine for two consecutive months. TB treatment success rate was $96.9 \%$ in intervention group and $94.26 \%$ in controls, $\mathrm{p}-0.983$

Limitations Didn't assess background knowledge of participants

Study ID Johnston, et al, 2018 [52]

Setting Canada

Methods A parallel, randomized controlled trial

Participants Adults initiating LTBI therapy between June 2012 and September 2015

Sample size Overall, the study enrolled 358 participants (170 in intervention and 188 in control arms)

Interventions An interactive (two-way) text and phone call message service for LTBI adherence.

Outcomes Treatment completion was 79\% in intervention and 82\% in control groups with RR 0.97; $p=0.550$

Limitations Outcome influenced by intensive monitoring schedule of the standard care

Study ID Georges B. et al, 2018 [53]

Setting Cameroon

Methods A randomized controlled trial conducted between February 2013 and April 2014

Participants Adults (> 18 years) and newly diagnosed PTB patients

Sample size Two hundred seventy-nine participants; 137 in intervention 142 in control groups

Interventions A one-way daily text-based reminder and motivational messages for six months

Outcomes At five months, treatment success was $81 \%$ in intervention and $75 \%$ in control groups with $\mathrm{OR}=1.45$; $p=0.203$.

Limitations High attrition of participants

Study ID Hermans SM. et al, 2017 [54]

Setting Uganda

Methods Quasi-experimental study design held between November 2010 and October 2011

Participants Adult, literate, HIV/TB patients access with mobile phone

Sample size Overall 485 (183 in intervention and 302 in control groups) followed up.

Interventions An interactive (two way) text-based medication and appointment reminder, and educational messages using a total of 8 SMSs per 2 weeks for two months

Outcomes After 8 weeks intervention, successful completion of treatment was $93 \%$ in intervention and $89 \%$ in control 
Table 3 Characteristics studies included in the review (Continued) groups, $\mathrm{p}-0.43$.

Limitations Use of pre-intervention control group prone to tempora changes could influence outcomes

Study ID Kumboyono, 2017 [48]

Setting Indonesia

Methods A post-test-only controlled-group design

Participants Adult TB patients enrolled on treatment

Sample size 45 TB patients enrolled on treatment

Interventions A text-based phone messaging to motivate patients

Outcomes There was no difference in treatment compliance between the SMS and control groups with a p-0.059 of Fisher's Exact test.

Limitations Limited sample size

Study ID Kunawararak et'al, 2011 [41]

Setting Thailand

Methods A two arm RCT between April 2008 and December 2009

Participants New sputum smear positive pulmonary TB patients (both non-MDR-TB and MDR-TB) Patients aged > 15 years

Sample size 98 (60 Non-MDR and 38 MDR) TB patients

Interventions An interactive daily phone call reminder for six and eighteen months

Outcomes Treatment success Rate (TSR) was significantly higher in intervention group (100\%) than control (96.7\%) in nonMDR-TB, (p-0.047).

Limitations Limited sample size

Study ID Liu et'al, 2012 [45]

Setting China

Methods

A pragmatic cluster-randomized trial in 36 districts. From 1 June 2011 to 7 March 2012, 4292 TB patients were enrolled across the clusters.

Participants New pulmonary TB patients, starting on standard 6month short-course chemotherapy

Sample size 4292 TB patients

Interventions An interactive daily text messages to reminder medications for six months

Outcomes TB treatment success was $96.1 \%$ in SMS groups, $91.4 \%$ in control groups, with $\mathrm{p}-0.084$

Limitations Over-estimation of poor adherence

Study ID Mohammed et'al, 2013 [43]

Setting Pakistan

Methods A two-arm, randomized controlled trial in Karachi, Pakistan. Individual participants were randomized to either SMS or the control group.

Participants Newly-diagnosed patients with smear or bacteriologically positive pulmonary tuberculosis who were on treatment for less than two weeks; $\geq 15$ years; reported having access to a mobile phone; and intended to live in Karachi throughout treatment were eligible. The study enrolled 2207 participants, with 1110 randomized to SMS and 1097 to the control group.

Sample size 2207 TB patients

Interventions An interactive daily text pill reminder for six months and
Table 3 Characteristics studies included in the review (Continued)

participants respond with SMS or missed calls after taking medication. Up to 3 SMSs sent for non-respondents a day.

Outcomes There was no significant difference between the SMS or control groups for treatment success (719 or $83 \%$ vs. 903 or $83 \%$, respectively, $p=0.782$ ).

Limitations Lack of an objective tool to measure adherence

Study ID Narasimhan et'al, 2013 [47]

Setting India

Methods Single arm interventional study

Participants TB patients seeking care from the DOTS centers

Sample size 104 patients recruited, 100 patients were followed until end of treatment

Interventions Text and/or voice call reminder enabled treatment adherence support system

Outcomes A voice call reminder system could improve patients adherence to TB drugs

Limitations The effect size of the intervention was not determined

Study ID Sarah I. et al, 2013 [42]

Setting Argentina

Methods A randomized 1:1 allocation

Participants Patients newly diagnosed with TB who were $\geq 18$ years, and had mobile-phone access

Sample size 38 TB patients (18 in intervention and 19 in control)

Interventions An interactive bi-weekly text-based educational messages to patients to adhere to medication for the first 2 months of treatment

Outcomes Treatment success was 17/18 in intervention arm and 17/ 19 in control arm.

Limitations Baseline knowledge not addressed; use self-reporting that may bias the outcome

analysis has shown that two-way phone messaging interventions have a small effect on TB treatment success as compared with one-way messaging. Evidence also indicated that SMS interventions for ART that run two-way SMS communication were more acceptable than non-interactive reminders [59]. An interactive communication could provide an opportunity for patients to get their voices heard, and improve relationships and enhance engagement in their treatment.

This systematic review has shown that mobile-phone messaging could be encouraging approach to improve Anti-TB treatment success, however, paucity of highquality evidence to direct policies. Detection, performance and attrition biases were observed in most of the studies included in this review.

The overall quality of evidence of this review was rated low by using GRADE approach. The main reasons were biases and low sample size in many of the studies. As a limitation, this systematic review and meta-analysis was 
reliant on inadequate number of studies. The review has merely included studies written in English.

\section{Conclusion}

This systematic review shown paucity of high-quality evidences concerning effect of mobile-phone messaging on anti-TB treatment success. Mobile-phone messaging showed a modest effect in improving anti-TB treatment success, however the quality of evidence was low. Further controlled studies are needed to increase the evidence-base on the role of mHealth interventions to improve TB care. This systematic review will be updated as new evidence emerged.

\section{Implications for practice}

Digital communication technologies including mHealth has promising impact on healthcare. The need to support further innovative mHealth initiatives from all stakeholders.

\section{Implications for future research}

Current evidence is of low quality. This implies that further well designed and reported RCT studies are needed for a better quality of evidence on Anti-TB treatment outcome.

\section{Supplementary information}

Supplementary information accompanies this paper at https://doi.org/10. 1186/s12879-020-4765-x

Additional file 1. The MeSH search terms used for systematic review and meta-analysis on effect of phone messaging on ant-Tb treatment outcome.

\section{Abbreviations \\ GRADE: Grading of Recommendations Assessment, Development and Evaluation; HBCs: High Burden Countries; MeSH: Medical Subject Headings; PICO: Population, Intervention, Comparison and Outcome; PRISMA: Preferred Reporting Items for Systematic Reviews and Meta-Analyses; RCT: Randomized Controlled Trial; SMS: Short Message Service; TB: Tuberculosis}

\section{Acknowledgments}

We acknowledge the authors for the original articles that were taken for pooled analysis. We are grateful to the Institute of Public Health, College of Medicine and Health Sciences, University of Gondar for direct and indirect support to undertake this review.

\section{Authors' contributions}

KDG, BT and KAG contributed to conception and design. KDG and ZAM participated in searching, selection and appraisal of articles. KDG, BT, KAG and ZAM involved in the analysis. KDG, BT, KAG and RL take part the interpretation and write-up. All authors approved the manuscript for publication.

\section{Funding}

The authors of the study covered all the budget.

\section{Availability of data and materials}

All the data were presented within the manuscript.

Ethics approval and consent to participate

Not applicable.
Consent for publication

Not applicable.

\section{Competing interests}

The authors declare that they have no competing interests.

\section{Author details}

${ }^{1}$ Department of Health Informatics, Institute of Public Health, College of Medicine and Health Sciences, University of Gondar, Gondar, Ethiopia. ${ }^{2}$ Department of Epidemiology and Biostatistics, Institute of Public Health, College of Medicine and Health Science, University of Gondar, Gondar, Ethiopia. ${ }^{3}$ Research Pavilion, Rm 566, 828 W 10th, University of British Columbia, Vancouver, BC V5Z 1 M9, Canada.

Received: 28 August 2019 Accepted: 6 January 2020

Published online: 14 January 2020

\section{References}

1. World Bank, Mobile cellular subscriptions, ITU, World Telecommunication/ ICT Development Report and database https://data.worldbank.org/indicator/ IT.CEL.SETS.P2. 2016.

2. ITU. ICT facts and figures. Geneva: International Telecommunications Union; 2010. http://www.itu.int/ITU-D/ict/material/FactsFigures2010.pdf, Accessed 1 Nov 2016

3. Anglada-Martinez $\mathrm{H}$, et al. Does mHealth increase adherence to medication? Results of a systematic review. Int J Clin Pract. 2015;69(1):9-32.

4. Lester RT, et al. Effects of a mobile phone short message service on antiretroviral treatment adherence in Kenya (WelTel Kenya1): a randomised trial. Lancet. 2010:376(9755):1838-45.

5. Chaiyachati $\mathrm{KH}$, et al. A pilot study of an mHealth application for healthcare workers: poor uptake despite high reported acceptability at a rural south African community-based MDR-TB treatment program. PLoS One. 2013;8(5): e64662.

6. Guy R, et al. How effective are short message service reminders at increasing clinic attendance? A meta-analysis and systematic review. Health Serv Res. 2012:47(2):614-32.

7. Govindasamy D, et al. Interventions to improve or facilitate linkage to or retention in pre-ART (HIV) care and initiation of ART in low- and middle-income settings--a systematic review. J Int AIDS Soc. 2014;17: 19032.

8. WHO, Resolution WHA67.1. Global strategy and targets for tuberculosis prevention, care and control after 2015. http://apps.who.int/gb/ebwha/pdf files/WHA67/A67_R1-en.pdf Date last Accessed: 9 Nov 2016.

9. WHO, Global Task Force on digital health for TB. www.who.int/tb/areas-ofwork/digital-health/global-task-force/en/ Accessed: 20 Nov 2016.

10. Fialkowski MK, et al. A community engagement process identifies environmental priorities to prevent early childhood obesity: the Children's healthy living $(\mathrm{CHL})$ program for remote underserved populations in the US affiliated Pacific Islands, Hawaii and Alaska. Matern Child Health J. 2014; 18(10):2261-74.

11. Actor JK, et al. Mycobacterial glycolipid cord factor trehalose 6,6'-dimycolate causes a decrease in serum cortisol during the granulomatous response. Neuroimmunomodulation. 2002;10(5):270-82.

12. WHO, Global tuberculosis report. 2016.

13. WHO. Global Tuberculosis Report 2014. Geneva: World Health Organization; 2014. URL: http://apps.who.int/iris/bitstream/10665/137094/1/9789241564 809 eng.pdf?ua $=1$

14. WHO, Global tuberculosis report 2018: https://www.who.int/tb/publications/ global_report/en/ Accessed 5 Oct 2019. 2018.

15. World Health Organization. Stop TB Dept. and World Health Organization. Dept. of HIV/AIDS. Interim policy on collaborative TB/HIV activities. Geneva: World Health Organization; 2004. p. 19.

16. Jensen PA, et al. Guidelines for preventing the transmission of Mycobacterium tuberculosis in health-care settings, 2005. MMWR Recomm Rep. 2005;54(RR-17):1-141.

17. WHO, Adherence to long-term therapies: evidence for action. 2003

18. Adane AA, et al. Non-adherence to anti-tuberculosis treatment and determinant factors among patients with tuberculosis in Northwest Ethiopia. PLoS One. 2013;8(11):e78791. 
19. Martin C, Perfect T, Mantle G. Non-attendance in primary care: the views of patients and practices on its causes, impact and solutions. Fam Pract. 2005; 22(2):38-43.

20. Neal RD, et al. Reasons for and Consequences of Missed Appointments in General Practice in the UK: Questionnaire Survey and Prospective Review of Medical Records. BMC Fam Pract. 2005;6:47.

21. van Baar JD, et al. Understanding reasons for asthma outpatient (non)attendance and exploring the role of telephone and e-Consulting in Facilitating Access to care: exploratory qualitative study. Qual Saf Health Care. 2006;15(3):191-5.

22. Crosby LE, et al. Perceived barriers to clinic appointments for adolescents with sickle cell disease. J Pediatr Hematol Oncol. 2009;31(8):571-6.

23. Liu Q, et al. Reminder systems to improve patient adherence to tuberculosis clinic appointments for diagnosis and treatment. Cochrane Database Syst Rev. 2014;11:CD006594.

24. Iribarren SJ, et al. Smartphone applications to support tuberculosis prevention and treatment: review and evaluation. JMIR Mhealth Uhealth. 2016;4(2):e25.

25. Nglazi MD, et al. Mobile phone text messaging for promoting adherence to anti-tuberculosis treatment: a systematic review. BMC Infect Dis. 2013;13:566.

26. World Health Organization. Stop TB Dept. Treatment of tuberculosis: guidelines. 4th ed. Geneva: World Health Organization; 2010. p. 147.

27. WHO. Global tuberculosis report 2018. Geneva: World Health Organization; 2018. Licence: CC BY-NC-SA 3.0 IGO. https://www.who.int/tb/publications/ global_report/en/. Accessed 26 Aug 2019

28. Higgins JPT, Green S. Cochrane handbook for systematic reviews of interventions version 5.0.2. Chichester: Wiley; 2009.

29. Cohen J. A coefficient of agreement for nominal scales. Educ Psychol Meas. 1960;20(1):37-46.

30. DerSimonian R, Laird N. Meta-analysis in clinical trials. Control Clin Trials. 1986;7(3):177-88.

31. Riley RD, Higgins JP, Deeks JJ. Interpretation of random effects metaanalyses. BMJ. 2011;342:d549.

32. Guyatt G, et al. GRADE guidelines: 1. Introduction-GRADE evidence profiles and summary of findings tables. J Clin Epidemiol. 2011;64(4):383-94.

33. Garfein RS, et al. Feasibility of tuberculosis treatment monitoring by video directly observed therapy: a binational pilot study. Int J Tuberc Lung Dis. 2015;19(9):1057-64.

34. Holzschuh EL, et al. Use of video directly observed therapy for treatment of latent tuberculosis infection - Johnson County, Kansas, 2015. MMWR Morb Mortal Wkly Rep. 2017;66(14):387-9.

35. Buchman T, Cabello C. A new method to directly observe tuberculosis treatment: Skype observed therapy, a patient-centered approach. J Public Health Manag Pract. 2017;23(2):175-7.

36. Hoffman JA, et al. Mobile direct observation treatment for tuberculosis patients: a technical feasibility pilot using mobile phones in Nairobi, Kenya. Am J Prev Med. 2010;39(1):78-80.

37. DeMaio J, et al. The application of telemedicine technology to a directly observed therapy program for tuberculosis: a pilot project. Clin Infect Dis. 2001;33(12):2082-4.

38. Lorent $\mathrm{N}$, et al. Community-based active tuberculosis case finding in poor urban settlements of Phnom Penh, Cambodia: a feasible and effective strategy. PLoS One. 2014;9(3):e92754.

39. Bassett IV, et al. Sizanani: a randomized trial of health system navigators to improve linkage to HIV and TB care in South Africa. J Acquir Immune Defic Syndr. 2016;73(2):154-60.

40. Howard AA, et al. The START study to evaluate the effectiveness of a combination intervention package to enhance antiretroviral therapy uptake and retention during TB treatment among TB/HIV patients in Lesotho: rationale and design of a mixed-methods, cluster-randomized trial. Glob Health Action. 2016;9(1):31543.

41. Kunawararak $P$, et al. Tuberculosis treatment with mobile-phone medication reminders in northern Thailand. Southeast Asian J Trop Med Public Health. 2011;42(6):1444-51.

42. Iribarren S, et al. TextTB: a mixed method pilot study evaluating acceptance, feasibility, and exploring initial efficacy of a text messaging intervention to support TB treatment adherence. Tuberc Res Treat. 2013; 2013:349394.

43. Mohammed S, Glennerster R, Khan AJ. Impact of a daily SMS medication reminder system on tuberculosis treatment outcomes: a randomized controlled trial. PLoS One. 2016;11(11):e0162944.
44. Farooqi RJ, Ashraf S, Zaman M. The role of mobile SMS-reminders in improving drugs compliance in patients receiving anti-TB treatment from DOTS program. https://www.jpmi.org.pk/index.php/jpmi/article/view/2037. Accessed 23 Aug 2019.

45. Liu X, et al. Effectiveness of electronic reminders to improve medication adherence in tuberculosis patients: a cluster-randomised trial. PLoS Med. 2015;12(9):e1001876.

46. Barclay E. Text messages could hasten tuberculosis drug compliance. Lancet. 2009;373(9657):15-6.

47. Narasimhan $P$, et al. A customized $m$-health system for improving tuberculosis treatment adherence and follow-up in South India. Health Technol. 2014;4(1):1-10. https://doi.org/10.1007/s12553-013-0067-2

48. Kumboyono. Short message service as an alternative in the drug consumption evaluation of persons with tuberculosis in Malang, Indonesia. Jpn J Nurs Sci. 2017;14(2):112-6. https://doi.org/10.1111/jjns.12140.

49. Belknap R, et al. Self-administered versus directly observed once-weekly isoniazid and Rifapentine treatment of latent tuberculosis infection: a randomized trial. Ann Intern Med. 2017;167(10):689-97. https://doi.org/10. 7326/M17-1150

50. bridges.org, Evaluation of the On Cue Compliance Service Pilot Testing the use of SMS reminders in the treatment of Tuberculosis in Cape Town, South Africa. 2005. https://healthmarketinnovations.org/sites/default/files/On\%2 0Cue\%20Compliance\%20Impact\%20Evaluation.pdf. Accessed 23 Aug 2019.

51. Fang XH, et al. Effect of short message service on Management of Pulmonary Tuberculosis Patients in Anhui Province, China: a prospective, randomized, controlled study. Med Sci Monit. 2017:23:2465-9. https://doi. org/10.12659/MSM.904957

52. Johnston JC, et al. The effect of text messaging on latent tuberculosis treatment adherence: a randomised controlled trial. Eur Respir J. 2018;51(2). https://doi.org/10.1183/13993003.01488-2017

53. Bediang $\mathrm{G}$, et al. SMS reminders to improve adherence and cure of tuberculosis patients in Cameroon (TB-SMS Cameroon): a randomised controlled trial. BMC Public Health. 2018;18(1):583.

54. Hermans SM, et al. Text messaging to decrease tuberculosis treatment attrition in TB-HIV coinfection in Uganda. Patient Prefer Adherence. 2017;11: 1479-87.

55. Getahun $\mathrm{H}$, et al. Management of latent mycobacterium tuberculosis infection: WHO guidelines for low tuberculosis burden countries. Eur Respir J. 2015;46(6):1563-76.

56. Denkinger $\mathrm{CM}$, et al. Mobile health to improve tuberculosis care and control: a call worth making. Int J Tuberc Lung Dis. 2013;17(6):719-27.

57. Falzon D, et al. Digital health for the end TB strategy: developing priority products and making them work. Eur Respir J. 2016;48(1):29-45.

58. Hall AK, Cole-Lewis H, Bernhardt JM. Mobile text messaging for health: a systematic review of reviews. Annu Rev Public Health. 2015;36:393-415.

59. Amankwaa I, et al. Effectiveness of short message services and voice call interventions for antiretroviral therapy adherence and other outcomes: a systematic review and meta-analysis. PLoS One. 2018;13(9):e0204091.

60. Ngwatu BK, et al. The impact of digital health technologies on tuberculosis treatment: a systematic review. Eur Respir J. 2018;51(1):1701596.

61. Nglazi MD, et al. Mobile phone text messaging for promoting adherence to anti-tuberculosis treatment: a systematic review. BMC Infect Dis. 2013;13:566.

62. Lester $\mathrm{R}$, et al. Mobile phone short message service for adherence support and care of patients with tuberculosis infection: evidence and opportunity. J Clin Tuberc Other Mycobact Dis. 2019;16:100108.

\section{Publisher's Note}

Springer Nature remains neutral with regard to jurisdictional claims in published maps and institutional affiliations. 